\title{
Generalized James' effective Hamiltonian method
}

\author{
Wenjun Shao, ${ }^{1}$ Chunfeng $\mathrm{Wu}^{2}$ and Xun-Li Feng ${ }^{1, *}$ \\ ${ }^{1}$ Department of Physics, Shanghai Normal University, Shanghai 200234, China \\ ${ }^{2}$ Pillar of Engineering Product Development, Singapore University \\ of Technology and Design, 8 Somapah Road, Singapore 487372
}

\begin{abstract}
James' effective Hamiltonian method has been extensively adopted to investigate largely detuned interacting quantum systems. This method is just corresponding to the second-order perturbation theory, and cannot be exploited to treat the problems which should be solved by using the thirdor higher-order perturbation theory. In this paper, we generalize James' effective Hamiltonian method to the higher-order case. Using the method developed here, we reexamine two examples published recently [Phys. Rev.Lett. 117, 043601 (2016), Phys. Rev A 92, 023842 (2015)], our results turn out to be the same as the original ones derived from the third-order perturbation theory and adiabatic elimination method respectively. For some specific problems, this method can simplify the calculating procedure, and the resultant effective Hamiltonian is more general.
\end{abstract}

\section{INTRODUCTION}

Generally it is hard to obtain exact analytical solutions for quantum systems, thus various approximation methods have been developed to deal with couplings between the quantum systems. For instance, perturbation theory [1], adiabatic elimination method [2, 3] and James' effective Hamiltonian method [4-6] have been utilized in the derivation of effective Hamiltonian in quantum mechanics. However, for some specific physical problems the former two methods are time consuming and the deriving procedure is tediously long. While James' effective Hamiltonian method may sometimes provide us an efficient tool and has been employed to solve many interesting questions related to light-matter interactions [7 9].

We have noted that the James' effective Hamiltonian method is actually corresponding to the second-order perturbation theory. For example, the effective Hamiltonian of the Mølmer and Sørensen scheme for trapped ions can be derived from both the second-order perturbation theory [10, 11] and the James' effective Hamiltonian method [4]. Accordingly in what follows we refer to the original James' effective Hamiltonian method as the second-order James' method.

Very recently, the third-order perturbation theory was exploited to deal with problems related to the ultrastrong coupling between the cavity field and atoms. For instance, by applying the third-order perturbation theory, a surprising result that one photon can simultaneously excite two or more atoms was deduced in the ultrastrong coupling regime with a symmetry-broken potential [12]. As the other example, when the frequency of the cavity field is near one-third of the atomic transition frequency, a resonant three-photon coupling between a twolevel atom and the cavity field can be constructed which was derived by the adiabatic elimination method in Law's group [3]. We find one can obtain the similar result by using the third-order perturbation theory. Unfortunately,

\footnotetext{
* xlfeng@shnu.edu.cn
}

one cannot derive the effective Hamiltonian appearing in Refs. [12] and [3] by using original James' effective Hamiltonian method, the reason is that James' method is only corresponding to the second-order perturbation theory as mentioned above. So it is necessary to generalize James' method to higher-order. This is the purpose of our present work.

The paper is organized as follows. In Sec. II, we give a simple review about James' effective Hamiltonian method. And in Sec. III, we derive the third- and $n$ th-order James' effective Hamiltonian by generalizing James' method. Then in Sec. IV we use the generalized James' method to derive the effective Hamiltonian in Refs. [12] and [3], and compare both results. Finally, a summary is made in Sec. V.

\section{REVIEW OF JAMES' EFFECTIVE HAMILTONIAN METHOD}

Now let us give a simple review about James' effective Hamiltonian method [5, 6], which is well suitable for treating strongly detuned interacting quantum systems, such as atomic or molecular interacting with laser beams, spin system interacting with oscillating magnetic fields under the conditions of highly detuned regime. In the interaction picture the system mentioned above is governed by the Hamiltonian, $\hat{H}_{I}(t)$, of the form

$$
\hat{H}_{I}(t)=\sum_{m}\left[\hat{h}_{m} \exp \left(i \omega_{m} t\right)+\hat{h}_{m}^{\dagger} \exp \left(-i \omega_{m} t\right)\right] .
$$

The Schrödinger equation is

$$
i \hbar \frac{\partial}{\partial t}|\psi(t)\rangle=\hat{H}_{I}(t)|\psi(t)\rangle .
$$

The formal solution to Eq. (2) is

$$
|\psi(t)\rangle=|\psi(0)\rangle+\frac{1}{i \hbar} \int_{0}^{t} \hat{H}_{I}\left(t^{\prime}\right)\left|\psi\left(t^{\prime}\right)\right\rangle d t^{\prime}
$$


Substituting Eq. (3) into Eq. (2) and neglecting highly oscillating terms $\hat{H}_{I}(t)|\psi(0)\rangle$ yield

$$
i \hbar \frac{\partial}{\partial t}|\psi(t)\rangle=\frac{1}{i \hbar} \hat{H}_{I}(t) \int_{0}^{t} \hat{H}_{I}\left(t^{\prime}\right)\left|\psi\left(t^{\prime}\right)\right\rangle d t^{\prime} .
$$

By Markovian approximation, Eq. (4) becomes

$$
i \hbar \frac{\partial}{\partial t}|\psi(t)\rangle=\hat{H}_{e f f}^{(2)}(t)|\psi(t)\rangle
$$

where

$$
\hat{H}_{\text {eff }}^{(2)}(t)=\frac{1}{i \hbar} \hat{H}_{I}(t) \int_{0}^{t} \hat{H}_{I}\left(t^{\prime}\right) d t^{\prime} .
$$

Here the superscript "(2)" indicates the second-order James' effective Hamiltonian as mentioned in Sec. I, which is corresponding to the second-order perturbation theory.

Substituting Eq. (1) into Eq. (6) and taking all frequencies $\omega_{m}$ distinct, we obtain the following form by using the rotating wave approximation

$$
\hat{H}_{e f f}^{(2)}(t)=\sum_{m} \frac{1}{\hbar \omega_{m}}\left[\hat{h}_{m}, \hat{h}_{m}^{\dagger}\right]
$$

Note that for the case that the frequencies $\omega_{m}$ are not all distinct, e.g., $\left|\omega_{m}-\omega_{n}\right| \ll \omega_{m}, \omega_{n}$, one should take into account the terms containing $\hat{h}_{m} \hat{h}_{n}^{\dagger} e^{i\left(\omega_{m}-\omega_{n}\right) t}$, $\hat{h}_{m}^{\dagger} \hat{h}_{n} e^{-i\left(\omega_{m}-\omega_{n}\right) t}$ as did in [5]. However, for simplicity here and in what follows we only consider the case of all the frequencies $\omega_{m}$ being distinct.

\section{GENERALIZED JAMES' EFFECTIVE HAMILTONIAN METHOD}

In order to develop a generalized James' method corresponding to the third- and higher-order perturbation theory, we adopt James' idea as mentioned in Sec. II. First of all, substituting Eq. (3) into Eq. (4) with iteration and yields

$$
\begin{aligned}
i \hbar \frac{\partial}{\partial t}|\psi(t)\rangle & =\left[\frac{1}{i \hbar} \hat{H}_{I}(t) \int_{0}^{t} \hat{H}_{I}\left(t_{1}\right) d t_{1}+\left(\frac{1}{i \hbar}\right)^{2} \hat{H}_{I}(t) \int_{0}^{t} \hat{H}_{I}\left(t_{1}\right) \int_{0}^{t_{1}} \hat{H}_{I}\left(t_{2}\right) d t_{2} d t_{1}+\cdots\right. \\
& \left.+\left(\frac{1}{i \hbar}\right)^{n-1} \hat{H}_{I}(t) \int_{0}^{t} \hat{H}_{I}\left(t_{1}\right) \int_{0}^{t_{1}} \hat{H}_{I}\left(t_{2}\right) \cdots \int_{0}^{t_{n-2}} \hat{H}_{I}\left(t_{n-1}\right) d t_{n-1} \cdots d t_{2} d t_{1}+\cdots\right]|\psi(0)\rangle,
\end{aligned}
$$

Taking Markovian approximation, we obtain

$$
i \hbar \frac{\partial}{\partial t}|\psi(t)\rangle=\hat{H}_{e f f}(t)|\psi(t)\rangle
$$

where

$$
\hat{H}_{e f f}(t)=\hat{H}_{\text {eff }}^{(2)}(t)+\hat{H}_{\text {eff }}^{(3)}(t)+\cdots+\hat{H}_{\text {eff }}^{(n)}(t)+\cdots,
$$

$\hat{H}_{\text {eff }}^{(2)}(t)$ is the second-order James' effective Hamiltonian expressed by Eq. (6) and $\hat{H}_{\text {eff }}^{(3)}(t)$ is referred to as the third-order James' effective Hamiltonian of the following form

$$
\hat{H}_{\text {eff }}^{(3)}(t)=-\frac{1}{\hbar^{2}} \hat{H}_{I}(t) \int_{0}^{t} \hat{H}_{I}\left(t_{1}\right) \int_{0}^{t_{1}} \hat{H}_{I}\left(t_{2}\right) d t_{2} d t_{1},
$$

$\hat{H}_{e f f}^{(n)}(t)$ is the $n$ th-order James' effective Hamiltonian

$$
\begin{aligned}
\hat{H}_{e f f}^{(n)}(t)= & \left(\frac{1}{i \hbar}\right)^{n-1} \hat{H}_{I}(t) \int_{0}^{t} \hat{H}_{I}\left(t_{1}\right) \int_{0}^{t_{1}} \hat{H}_{I}\left(t_{2}\right) \times \\
& \cdots \int_{0}^{t_{n-2}} \hat{H}_{I}\left(t_{n-1}\right) d t_{n-1} \cdots d t_{2} d t_{1} .
\end{aligned}
$$

Examining our results (10)-(12), one can find the effective Hamiltonian in arbitrary orders is actually equivalent to the series expansion of the unitary evolution operator $U(t, 0)[13,14]$. To this end, now let us briefly derive the series expansion of the unitary evolution operator from Eq. (8). The formal solution to Eq. (8) is

$$
\begin{aligned}
|\psi(t)\rangle & =\left[1+\frac{1}{i \hbar} \int_{0}^{\tau} \hat{H}_{\text {eff }}(t) d t\right]|\psi(0)\rangle \\
& =U(t, 0)|\psi(0)\rangle,
\end{aligned}
$$


where

$$
\begin{gathered}
U(t, 0)=1+\sum_{n=1}^{\infty}\left(\frac{1}{i \hbar}\right)^{n} \int_{0}^{\tau} \hat{H}_{I}(t) \int_{0}^{t} \hat{H}_{I}\left(t_{1}\right) \times \\
\cdots \int_{0}^{t_{n-2}} \hat{H}_{I}\left(t_{n-1}\right) d t_{n-1} \cdots d t_{1} d t .
\end{gathered}
$$

It is not difficult to check that $U(t, 0)$ in Eq. (14) is exactly the series expansion of the unitary evolution operator [13, 14]. Different from the method of series ex- pansion of the unitary evolution operator, the generalized James' method developed here can give directly the effective Hamiltonian in arbitrary order.

In what follows we mainly focus on the third-order case and limit ourselves to the case that all of the frequencies $\omega_{m}$ are not only distinct, but also the algebraic sum of any three frequencies including two same ones is zero or distinct from zero. Considering $\hat{H}_{I}(t)$ taking the form of Eq. (1), one can further simplify $\hat{H}_{e f f}^{(3)}(t)$ by using the rotating wave approximation,

$$
\begin{aligned}
\hat{H}_{\text {eff }}^{(3)}(t) & =\frac{1}{\hbar^{2}} \sum_{l, m, n}\left\{\frac { 1 } { \omega _ { n } ( \omega _ { n } - \omega _ { m } ) } \left[\hat{h}_{l} \hat{h}_{m}^{\dagger} \hat{h}_{n} e^{i\left(\omega_{l}-\omega_{m}+\omega_{n}\right) t}+\hat{h}_{l}^{\dagger} \hat{h}_{m} \hat{h}_{n}^{\dagger} e^{i\left(-\omega_{l}+\omega_{m}-\omega_{n}\right) t}+\hat{h}_{l} \hat{h}_{m} \hat{h}_{n}^{\dagger} e^{i\left(\omega_{l}+\omega_{m}-\omega_{n}\right) t}\right.\right. \\
& \left.\left.+\hat{h}_{l}^{\dagger} \hat{h}_{m}^{\dagger} \hat{h}_{n} e^{i\left(-\omega_{l}-\omega_{m}+\omega_{n}\right) t}\right]+\frac{1}{\omega_{n}\left(\omega_{n}+\omega_{m}\right)}\left[\hat{h}_{l}^{\dagger} \hat{h}_{m} \hat{h}_{n} e^{i\left(-\omega_{l}+\omega_{m}+\omega_{n}\right) t}+\hat{h}_{l} \hat{h}_{m}^{\dagger} \hat{h}_{n}^{\dagger} e^{i\left(\omega_{l}-\omega_{m}-\omega_{n}\right) t}\right]\right\} .
\end{aligned}
$$

Note that in the above equation only the terms with sum frequency $\omega_{l}+\omega_{m}+\omega_{n}$ have been neglected. Since the frequencies $\omega_{m}$ are all distinct, one needs only to keep the terms in $\hat{H}_{e f f}^{(3)}$ from the contributions of algebraic sum of any three frequencies being zero and other contributions are all neglected according to rotating wave approximation. That is to say, Eq. (15) can be further simplified according to the practical situation. After such simplification, it is not difficult to prove $\hat{H}_{e f f}^{(3)}$ is hermitian, which is provided in appendix.

\section{EXAMPLES}

In this section, we reexamine two examples in Refs. [12] and [3] by the generalized James' method. As the first example, we examine two (or more) atoms excited simultaneously by one photon and compare the results with the original ones from the third perturbation theory in [12]. Then we revisit three-photon absorption of a twolevel atom via the counter-rotating processes in the Rabi model [3].

\section{A. Two atoms excited simultaneously by one photon}

In a recent literature an interesting result that two or more atoms can be simultaneously excited by one photon was derived in the ultrastrong coupling regime with a symmetry-broken potential [12]. The system considered in 12] is two or more identical qubits coupling to a single cavity mode. In the following we apply the generalized James' method to revisit this question in the case of two atoms. In the interaction picture with respect to $\hat{H}_{0}=\frac{1}{2} \omega_{q} \sum_{i=1,2} \hat{\sigma}_{z}^{i}+\omega_{c} a^{\dagger} a$ the Hamiltonian is given by $(\hbar=1)$

$$
\begin{aligned}
\hat{H}_{I} & =\sum_{i=1,2} \lambda \hat{a}^{\dagger}\left[\cos \theta\left(\hat{\sigma}_{-}^{i} e^{i \omega_{q} t}+\hat{\sigma}_{+}^{i} e^{3 i \omega_{q} t}\right)\right. \\
& \left.+\sin \theta \hat{\sigma}_{z}^{i} e^{2 i \omega_{q} t}\right]+H . c
\end{aligned}
$$

where $\omega_{c}\left(\omega_{q}\right)$ is the resonant frequency of the cavity mode (the qubit transition frequency), $\hat{\sigma}_{x}^{i}$ and $\hat{\sigma}_{z}^{i}$ are Pauli operators for the $i$ th qubit, $a^{\dagger}(a)$ is the photon creation (annihilation) operator for cavity mode, and $\lambda$ is the coupling strength of each qubit to the cavity mode. Note that to get Eq. (16) we have taken $\omega_{c}=2 \omega_{q}$. According to Eq. (1), $\hat{H}_{I}$ expressed in Eq. (16) possesses three distinct frequencies, $\omega_{1}=\omega_{q}, \omega_{2}=2 \omega_{q}$ and $\omega_{3}=$ $3 \omega_{q}$ and the corresponding $\hat{h}_{m}(m=1,2,3)$ are of the form

$$
\begin{aligned}
& \hat{h}_{1}=\lambda \sum_{i=1,2} \cos \theta \hat{a}^{\dagger} \hat{\sigma}_{-}^{i}, \\
& \hat{h}_{2}=\lambda \sum_{i=1,2} \sin \theta \hat{a}^{\dagger} \hat{\sigma}_{Z}^{i}, \\
& \hat{h}_{3}=\lambda \sum_{i=1,2} \cos \theta \hat{a}^{\dagger} \hat{\sigma}_{+}^{i} .
\end{aligned}
$$

By utilizing the third-order James' effective Hamiltonian method, we arrive at

$$
\hat{H}_{e f f}^{(3)}=-\frac{8 \lambda^{3} \cos ^{2} \theta \sin \theta}{3 \omega_{q}^{2}}\left(\hat{a}^{\dagger} \hat{\sigma}_{-}^{1} \hat{\sigma}_{-}^{2}+\hat{a} \hat{\sigma}_{+}^{1} \hat{\sigma}_{+}^{2}\right) .
$$

First of all, let us assume the initial state of the system is $|g g 1\rangle$ as did in Ref. [12], where $|g g 1\rangle \equiv|g\rangle_{1} \otimes|g\rangle_{2} \otimes|1\rangle_{C}$ standing for both atoms are in their ground state and the cavity mode in the one photon Fock state. In this case $\hat{H}_{\text {eff }}^{(3)}$ turns out to be the same as that in Ref. [12]. 
Obviously, our method is much simpler than the third perturbation theory used in [12].

Moreover, Eq. (17) shows richer physical connotation if one considers the initial state of the cavity mode in Fock state $|n\rangle_{C}$ and both atoms are still in their ground state, in such a case, Eq. (17) can be written as

$$
\hat{H}_{e f f}^{(3)}=\Omega_{e f f}^{(3)}(|e e(n-1)\rangle\langle g g n|+| g g n\rangle\langle e e(n-1)|),
$$

where $\Omega_{e f f}^{(3)}=-\frac{8 \sqrt{n} \lambda^{3} \cos ^{2} \theta \sin \theta}{3 \omega_{q}^{2}}$ is the effective Rabi frequency, and it is proportional to $\sqrt{n}$. That is to say, more photons in the cavity can enhance the ability for one photon to simultaneously excite two atoms.

\section{B. Three-photon coupling in the large-detuned Rabi model}

In the recent contribution, Ma and Law investigated theoretically the three-photon coupling in Rabi model in the large-detuning regime, they found a two-level atom can absorb three photons simultaneously via the counterrotating processes in the three-photon resonance [3]. In their study, the adiabatic elimination method was used to derive the effective Hamiltonian. Here we show the generalized James' method is also available to get the same result.

In the interaction picture with respect to $\hat{H}_{0}=$ $\frac{1}{2} \omega_{a} \hat{\sigma}_{z}+\omega_{c} \hat{a}^{\dagger} \hat{a}$ the Hamiltonian of the quantum Rabi model $(\hbar=1)$ is given by

$$
\hat{H}_{I}=\lambda\left[\hat{a} e^{i\left(\omega_{a}-\omega_{c}\right) t}+\hat{a}^{\dagger} e^{i\left(\omega_{a}+\omega_{c}\right) t}\right] \hat{\sigma}_{+}+H . c,
$$

where $\lambda$ is the coupling rate of atom to cavity mode, $\hat{a}$ and $\hat{a}^{\dagger}$ are, respectively, the annihilation and creation operators for the cavity field of frequency $\omega_{c}$, and $\omega_{a}$ is the atom transition frequency. The Pauli matrices are defined as $\hat{\sigma}_{z}=|e\rangle\langle e|-| g\rangle\left\langle g\left|, \hat{\sigma}_{+}=\right| e\right\rangle\left\langle g\left|, \hat{\sigma}_{-}=\right| g\right\rangle\langle e|$ and $\hat{\sigma}_{x}=\hat{\sigma}_{+}+\hat{\sigma}_{-}$. Under the three-photon resonance with $\omega_{c}=\omega_{a} / 3$, the interaction Hamiltonian becomes

$$
\hat{H}_{I}=\lambda \hat{\sigma}_{+}\left(\hat{a} e^{2 i \omega_{c} t}+\hat{a}^{\dagger} e^{4 i \omega_{c} t}\right)+H . c .
$$

Making the identification $\hat{h}_{1}=\lambda \hat{a} \hat{\sigma}_{+}$with frequency $\omega_{1}=2 \omega_{c}, \hat{h}_{2}=\lambda \hat{a}^{\dagger} \hat{\sigma}_{+}$with frequency $\omega_{2}=4 \omega_{c}$, one can straightforwardly utilize the formula expressed in Eq. (10) to find the effective Hamiltonian

$$
\begin{aligned}
& \hat{H}_{e f f}^{(2)}=\frac{\lambda^{2}}{4 \omega_{c}}\left[\left(3 \hat{a}^{\dagger} \hat{a}+2\right) \hat{\sigma}_{+} \hat{\sigma}_{-}-\left(3 \hat{a}^{\dagger} \hat{a}+1\right) \hat{\sigma}_{-} \hat{\sigma}_{+}\right] \\
& \hat{H}_{e f f}^{(3)}=-\frac{\lambda^{3}}{4 \omega_{c}^{2}}\left[\left(\hat{a}^{\dagger}\right)^{3} \hat{\sigma}_{-}+\hat{a}^{3} \hat{\sigma}_{+}\right] .
\end{aligned}
$$

If we take the specific state $|g, 3\rangle$ as the initial state of the system as did in Ref. [3] and consider in the same picture, say, interaction picture, the Eqs. (21) and (22) turn out to be the same as the result obtained in [3]. Moreover, if we take $|g, n\rangle$ as the initial state, the Eqs. (21) and (22) can give a more general result.

\section{SUMMARY}

In this work, we have generalized James' effective Hamiltonian method which corresponds to the secondorder perturbation theory to the case corresponding to the third- and higher-order perturbation theory, and we have shown that the effective Hamiltonian in arbitrary orders developed is actually equivalent to the series expansion of the unitary evolution operator. By using the generalized James' effective Hamiltonian method, we have reexamined two examples [3, 12] published recently, the resultant Hamiltonians are the same as the original ones derived from the third-order perturbation theory and adiabatic elimination method respectively. The generalized James' effective Hamiltonian method developed here can not only simplify the calculating procedure for some problems, but also provide us richer and more general results. We hope the generalized James' effective Hamiltonian method can be applicable to solve more quantum questions.

\section{ACKNOWLEDGMENT}

We are grateful to Yu Sixia for the helpful discussion and to the anonymous reviewer for the constructive suggestions and providing the Ref. [13]. This work is supported by the Natural Science Foundation of Shanghai (Grant No. 15ZR1430600), National Natural Science Foundation of China under Grant Nos. 61475168, 11674231 and 11074079. XLF is sponsored by Shanghai Gaofeng \& Gaoyuan Project for University Academic Program Development.

\section{APPENDIX: PROVING HERMITICITY OF $\hat{H}_{e f f}^{(3)}$}

In this appendix we will prove the hermiticity of $\hat{H}_{e f f}^{(3)}$. For simplicity, we concentrate our attention on the case, as mentioned in the text, that all of the frequencies $\omega_{m}$ are distinct, and the algebraic sum of any three frequencies is zero or distinct from zero, which includes both of the three frequencies are the same. Under such conditions, $\hat{H}_{e f f}^{(3)}$ only contains the terms from the contributions of algebraic sum of any three frequencies being zero and other contributions are all neglected according to rotating wave approximation. Apparently it is sufficient to prove one of such terms is hermitian. Without loss of generality, we suppose $\omega_{l}, \omega_{m}$ and $\omega_{n}$ are such three frequencies satisfying $\omega_{l}+\omega_{m}-\omega_{n}=0$, their contribution in $\hat{H}_{e f f}^{(3)}$ is set to $V_{l m n}, V_{l m n}$ can be simplified by using $\omega_{l}=\omega_{n}-\omega_{m}$ 


$$
V_{l m n}=\frac{1}{\hbar^{2}}\left[\frac{\hat{h}_{n}^{\dagger} \hat{h}_{l} \hat{h}_{m}+\hat{h}_{n} \hat{h}_{l}^{\dagger} \hat{h}_{m}^{\dagger}}{\omega_{n} \omega_{m}}+\frac{\hat{h}_{m} \hat{h}_{n}^{\dagger} \hat{h}_{l}+\hat{h}_{m}^{\dagger} \hat{h}_{n} \hat{h}_{l}^{\dagger}}{\omega_{m}\left(\omega_{m}-\omega_{n}\right)}+\frac{\hat{h}_{l} \hat{h}_{m} \hat{h}_{n}^{\dagger}+\hat{h}_{l}^{\dagger} \hat{h}_{m}^{\dagger} \hat{h}_{n}}{\omega_{n}\left(\omega_{n}-\omega_{m}\right)}\right]+\text { H.c. }
$$

It is not difficult to find that Eq. (20) is hermitian. For the case of $-\omega_{l}-\omega_{m}+\omega_{n}=0$, we can also prove its hermiticity. If two of the three frequencies are the same, say, $\omega_{l}=\omega_{m}$, one can prove this is just a spe- cial case. Therefore, we can ensure that the third-order James' effective Hamiltonian $\hat{H}_{\text {eff }}^{(3)}$ is hermitian.
[1] L. D. Landau, E. M. Lifshitz, Quantum Mechanics: Non-Relativistic Theory (Butterworth-Heinemann, 1981), Chap. 6.

[2] L. Wang, R. R. Puri, and J. H. Eberly, Coupled-channel cavity QED model and exact solutions, Phys. Rev. A 46, 7192 (1992).

[3] Ken K. W. Ma and C. K. Law, Three-photon resonance and adiabatic passage in the large-detuning Rabi model, Phys. Rev A 92, 023842 (2015).

[4] D. F. V. James, Quantum Computation with Hot and Cold Ions: An Assessment of Proposed Schemes, Fortschr. Phys. 48, 823 (2000).

[5] D. F. V. James, J. Jerke, Effective Hamiltonian Theory and Its Applications in Quantum Information, Can. J. Phys. 85, 625 (2007).

[6] O. Gamel and D. F. V. James, Time-averaged quantum dynamics and the validity of the effective Hamiltonian model, Phys. Rev. A 82, 052106 (2010).

[7] A. Sørensen and K. Mølmer, Entangling atoms in bad cavities, Phys. Rev. A 66, 022314 (2002).
[8] M. J. Hartmann, F. G. S. L. Brandao, and M. B. Plenio, Effective Spin Systems in Coupled Microcavities, Phys. Rev. Lett. 99, 160501 (2007).

[9] X. L Feng, C. Wu, H. Sun, and C. H. Oh, Geometric Entangling Gates in Decoherence-Free Subspaces with Minimal Requirements, Phys. Rev. Lett. 103, 200501 (2009).

[10] A. Sørensen and K. Mølmer, Quantum Computation with Ions in Thermal Motion, Phys. Rev. Lett. 82, 1971 (1999).

[11] K. Mølmer and A. Sørensen, Multiparticle Entanglement of Hot Trapped Ions, Phys. Rev. Lett. 82, 1835 (1999).

[12] L. Garziano, V. Macr, R. Stassi, O. Di Stefano, F. Nori, and S. Savasta, One Photon Can Simultaneously Excite Two or More Atoms, Phys. Rev. Lett. 117, 043601 (2016).

[13] A. L. Fetter and J. D. Waleka, Quantum theory of manyparticle systems (New York, 1971), Chap. 3, Eq. (6.19).

[14] W. H. Louisell, Quantum Statistical Properties of Radiation (New York, 1990), Chap. 1, Eq. (1.16.23). 